\title{
Shape effects on memory for location
}

\author{
Douglas H. Wedell, Sylvia Fitting, and Gary L. Allen \\ University of South Carolina, Columbia, South Carolina
}

\begin{abstract}
The participants were 40 students who were briefly presented 32 dot locations, one at a time, and attempted to reproduce each location after a short delay. Half of the participants completed the task with the surrounding shapes being a circle, a horizontal ellipse, and a vertical ellipse; for the other half, the surrounding shapes were a square, a triangle, and a pentagon. Elongation of the task field along an axis led to exaggerated bias along that axis, but the pattern of bias was fairly constant across the shapes. The data were modeled by assuming that bias in estimation was due to the weighting of spatial category prototypes. Modeling indicated that shape affected spacing of prototypes, but there was no evidence that it affected the number of prototypes. These results were consistent with use of a viewer-based frame of reference, with prototypes reflecting four spatial quadrants generated by left-right and up-down distinctions from the viewer's perspective.
\end{abstract}

There is abundant evidence that spatial information is coded in memory at both a fine-grain level that reflects coordinate-like memory and a coarser, categorical level that reflects how the space is partitioned (Huttenlocher, Hedges, \& Duncan, 1991; Spencer \& Hund, 2002). One way these two representations may be related is that the frame of reference guiding the orientation of coordinate memory may determine the categorization scheme. A viewer-based frame of reference implies partitioning the space into categories reflecting left-right, up-down, and front-back directions. A cue-based frame of reference implies categories centered on salient landmarks, such as when one describes a car as being close to a clock tower. A shape-based frame of reference implies categories that reflect a natural partitioning of the geometric shape of the environment, such as when one describes a point as being in the lower-left corner of a rectangle. The focus of the present research was to determine how simple manipulations of shape may influence the categorical encoding of spatial information in memory. The participants reproduced point locations within different shapes, and we modeled the bias in their estimates. Before describing our predictions, we first explain how shape may be used to develop a categorical representation of the spatial field, and then describe the modeling procedures we used for inferring that categorical representation.

It seems likely that geometric features of the environment may be used to determine spatial categories that serve to encode spatial locations. This supposition is bolstered by studies with animals and young toddlers, which have demonstrated a tendency for spatial localization to be based primarily on geometric features of the environment, largely ignoring environmental cues (Cheng, 1986; Hermer \& Spelke, 1994). Huttenlocher et al. (1991) provided evidence that when locating a dot in a circular field, individuals tend to bias their estimates toward a central location within the quadrant in which the dot appeared. According to their category-adjustment model, spatiallocation information is coded at a fine-grain level, which corresponds to its exact location within a coordinate system, and at a categorical level, which indicates into which quadrant the dot falls. As uncertainty about the location coded in fine-grain memory grows, the individual increasingly relies on categorical memory, so that estimates are biased toward the category prototype, which is assumed to be a centrally located value within the spatial category.

Extant research, however, has not yet determined how the category structure may depend on the geometric properties of the task field in this simple spatial-location task. Under the assumption that prototypes represent the central tendency of a geometric category, one would expect that stretching the geometric figure along vertical or horizontal axes should have a similar effect on the locations of category prototypes; that is, their locations along the axis of elongation will shift accordingly. On the other hand, if category prototypes are primarily determined by locations of targets within the field, which constitute exemplar information, then stretching the geometric figure should have no effect on locations of prototypes, as long as the distribution of target locations remains fixed.

We tested for the influence of shape on locations of prototypes using the six shapes shown in Figure 1, along with the locations of the 32 targets used for each shape. The circle and square shapes have been most extensively utilized and studied in the dot-location experimental paradigm (e.g., Fitting, Wedell, \& Allen, 2005; Huttenlocher et al., 1991; Spencer \& Hund, 2002). We transformed the circular shape along horizontal or vertical axes to create two different elliptical task fields. If prototypes are centrally located within a geometric region, then we would 


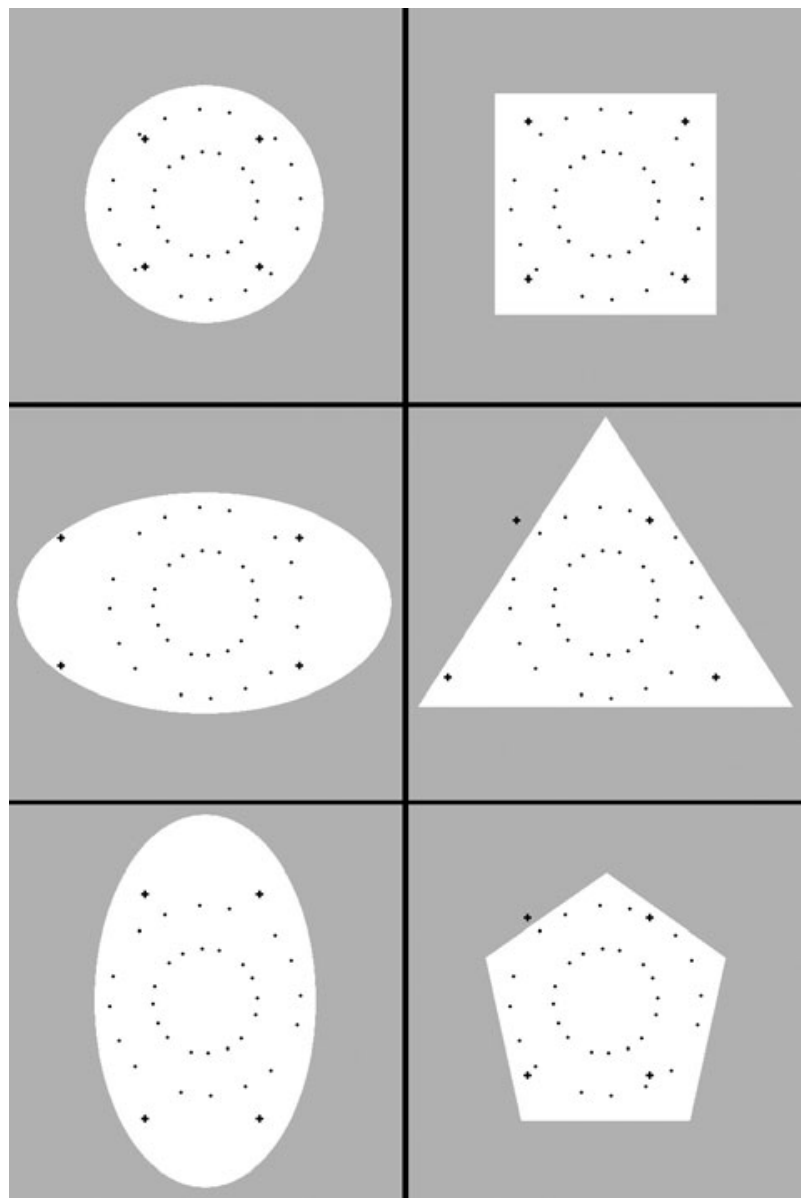

Figure 1. The six different shapes of the task fields used, along with the 32 dot locations for each. Crosses indicate inferred locations of category prototypes for each shape, generated from model fits to the data (see Discussion).

expect prototypes to have more extreme locations along the $x$-axis for the horizontal ellipse and more extreme locations along the $y$-axis for the vertical ellipse, in comparison with the prototype locations for the circle. On the other hand, if prototypes represent central tendencies within the distribution of target exemplars, no shifts in prototypes would be expected.

One group of participants made estimates of the 32 targets for each of the three curved shapes (left column of Figure 1) as a within-subjects test of the effects of stretching a figure along horizontal or vertical axes. The other group estimated spatial locations of the 32 targets for each of the three angular shapes (square, triangle, and pentagon) shown in the right column of Figure 1. Although there is some elongation of shapes in this condition, in that the base of the triangle is much wider than the bases of the square or pentagon, the primary purpose of this manipulation was to see whether number of prototypes might depend on number of corners or sides presented within the shape. If participants use an object-based, or allocentric, frame of reference, then the quadrant representation that is typically found for the square task field may be due to the four corners of the configuration supporting four spatial categories. If so, one would expect that the three corners of the triangle would support three spatial prototypes, and the five corners of the pentagon would support five spatial prototypes. On the other hand, Fitting et al. (2005) have speculated that the four prototypes typically found in this task arise from a viewer-centered frame of reference. From the viewer's perspective, the dot locations may be coded as up or down and right or left, combining into four spatial categories or regions. If spatial categories are viewer based, one would not expect increases in the number of prototypes with increases in the number of corners or edges of the shape. Rather, one would predict four prototypes for all six shapes shown in Figure 1, but with the locations of these prototypes likely influenced by elongation along the different axes.

The predictions we have described all concern how shape influences spatial category prototypes, either in their number or their locations. However, prototypes are not directly observed; rather, they are inferred from the pattern of bias in estimation. Our model for inferring prototype locations builds on the category-adjustment model of Huttenlocher et al. (1991), but deviates from it in some particulars. First, we adopt a fuzzy-boundary version of the model, similar to those versions described by Haun, Allen, and Wedell (2005) for angular bias in azimuth and elevation and by Fitting et al. (2005) for angular bias in the dot-location task. The fuzzy-boundary concept derives from the finding that locations near the border of two categories often show attenuated bias relative to what is predicted by the sharp-boundary model of Huttenlocher et al. (1991). The fuzzy boundaries account for this attenuation by assuming that prototypes are recruited on the basis of proximity of the target to the prototype. Locations near the boundary tend to recruit more than one prototype; the influences of these different prototypes tend to offset, resulting in reduced bias near the borders. A second way in which the model we used differed from previous models is that rather than adopt polar coordinates, we apply the model directly to the $x$ - and $y$-coordinates. There were three primary reasons for this choice. First, fitting the polar coordinates requires separate fits for angular bias (in degrees) and radial bias (in pixels), whereas fitting the Cartesian coordinates allows for a simultaneous fit of the two dimensions ( $x$ and $y$ ). Second, if the expected shifts in prototypes with elongation occur, then fitting radial bias becomes quite complex, because it depends on angular location. Finally, the polar coordinate approach implies greater error variance for longer radius targets, but this effect on variance was not observed.

Our basic model describes the mean response for stimulus $i$ along dimension $m$ as a weighted average of the actual location on that dimension (assumed to be the mean of the fine-grain memory representation) and the weighted sum of prototype locations along that dimension, as follows:

$$
R_{i m}=w S_{i m}+(1-w) \Sigma r_{i j} P_{j m},
$$


where $R_{i m}$ is the mean response for location $i$ on dimension $m, w$ is the relative weight of fine-grain memory, $S_{i m}$ is the actual stimulus location on dimension $m$ (which takes two values in this case, $x$ and $y), P_{j m}$ is the location of prototype $j$ on dimension $m$, and $r_{i j}$ is the relevance weight of prototype $j$ for stimulus $i$. Note that the fuzzy-boundary notion is captured by intermediate relevance weightings of prototypes, so that there may be more than one relevant prototype, especially as the target approaches the border between adjacent categories. In our model, relevance weighting is based on the similarity of the location to the prototype, where similarity is assumed to be a negative exponential function of Euclidean distance:

$$
r_{i j}=\frac{\exp \left\{-c\left[\left(S_{i x}-P_{j x}\right)^{2}+\left(S_{i y}-P_{j y}\right)^{2}\right]^{\frac{1}{2}}\right\}}{\sum \exp \left\{-c\left[\left(S_{i x}-P_{k x}\right)^{2}+\left(S_{i y}-P_{k y}\right)^{2}\right]^{\frac{1}{2}}\right\}},
$$

where $c$ is a sensitivity parameter that indicates how sharp the boundaries are and $r_{i j}$ indicates the proportionate influence of $P_{j}$ on the estimate of stimulus $i$, prior to weighting by $(1-w)$. Our modeling strategy was to combine Equations 1 and 2 and fit the $x$ and $y$ responses simultaneously after placing different constraints on number and locations of prototypes. To examine the goodness of fit of the model, we use the predicted values to residualize the responses and determine whether the key significant effects from the ANOVA are reduced or rendered nonsignificant once model predictions are taken into account. We also evaluated whether additional parameters significantly increased the variance explained for nested models.

\section{METHOD}

\section{Participants and Design}

A total of 40 undergraduate students from the University of South Carolina voluntarily participated in the experiment and received course credit for participation. Half were randomly assigned to the rounded shape condition and half to the angular shape condition.

In addition to the between-subjects variable of shape condition, five variables were manipulated within participants. The first of these was shape, manipulated at three levels: circle, horizontal ellipse, and vertical ellipse for the rounded set; and square, triangle, and pentagon for the angular set. The factorial combination of three variables, quadrant (4), radius (2, short and long), and angle (4), created the 32 targets presented for each shape. The final within-subjects variable was replication ( 2 replicates of each). The dependent variables were the $x$ - and $y$-coordinates of the location estimates, in pixels.

\section{Stimuli and Apparatus}

Microprocessors with 17-in. screens were used to present all of the experimental materials, with graphics presented in a $640 \times$ 480 pixel array. As shown in Figure 1, 32 target locations were presented for each shape, with locations generated from a polar coordinate system. Half of the targets were located at a short radius of 63 pixels and half at a long radius of 114 pixels. In each quadrant, targets were located at angles of $3^{\circ}, 25^{\circ}, 43^{\circ}$, and $75^{\circ}$. Dots marking the locations of targets, 4 pixels in diameter, were drawn in red. The areas of the shapes, in pixels, were as follows: circle, 61,575; ellipse, 91,232; square, 68,644; triangle, 76,590; and pentagon, 40,900. The key determinant of the size of the shapes was that it must accommodate the 32 target locations fairly closely within its borders.

\section{Procedure}

Groups of 1-5 participants were tested in a laboratory room, with computers spaced approximately $1 \mathrm{~m}$ apart. After reading general instructions, the participants were presented 3 demonstration trials, 1 for each of the three different shapes. Demonstration trials included feedback indicating the actual location. These were followed by 6 practice trials, 2 for each shape, with no feedback given, and then 192 randomly ordered experimental trials, in which the 32 targets for each of the three shapes were presented twice.

Each trial began with a 1-sec "ready" prompt followed by a 1.5sec blank screen. The target dot was then presented for $1 \mathrm{sec}$ on the screen inside the white task field for the given shape, with the rest of the screen in black. This was followed by a 4.5 -sec dynamic grayand-white checkerboard mask, contoured to the shape. The checkerboard consisted of squares (10 pixels wide), and the pattern shifted alternatively left and right by 10 pixels every $250 \mathrm{msec}$, to interfere with eye fixations. After the mask, a crosshair cursor appeared at the center of the white task field for the given shape. The participant moved the crosshairs using the mouse and clicked a mouse button to indicate the dot location.

\section{RESULTS}

The $x$ - and $y$-coordinates of the reproduced dot locations, averaged across replicates, constituted the dependent variable. An observed value was designated an outlier if its $x$-value or $y$-value was more than $2 S D$ s from the mean of responses for that location and shape condition. If both replicates of a point for a given participant were deemed outliers, we replaced the missing data point by the mean of the remaining values for the specific dot location within that condition. Less than $1 \%$ of data points were replaced.

To determine whether shape leads to systematic deviations of estimates from actual locations, we conducted separate repeated measures ANOVAs on bias scores for the two shape sets. We calculated bias scores by subtracting the actual $x$ - or $y$-value from the corresponding response. The within-subjects variables in each $2 \times 3 \times 4 \times 4 \times 2$ analysis were dimension, shape, quadrant, angle, and radius. Given the large number of tests and the replacement of some of the data by means, we adopted an alpha of .01 as a significance criterion.

Because the main focus of these studies was the influence of shape, we report only on the significant interactions involving shape. For the ANOVA on rounded shapes, there were five such significant interactions: shape $X$ quadrant $[F(6,114)=35.1]$; shape $\times$ dimension $\times$ quadrant $[F(6,114)=37.3]$; shape $\times$ quadrant $\times$ angle $[F(18,342)=8.2]$; shape $\times$ dimension $\times$ radius $\times$ quadrant $[F(6,114)=4.7]$; and shape $\times$ dimension $\times$ quadrant $X$ angle $[F(18,342)=7.4]$. For the ANOVA on angular shapes, there were four such significant interactions: shape $\times$ quadrant $[F(6,114)=11.0]$; shape $\times$ dimension $\times$ quadrant $[F(6,114)=21.6]$; shape $\times$ quadrant $\times$ angle $[F(18,342)=4.8]$; and shape $\times$ dimension $\times$ quadrant $\times$ angle $[F(18,342)=5.7]$.

To better understand this complex set of interactions, we modeled the data for the rounded shape and angular shape conditions separately, using Equations 1 and 2 with 
various constraints on parameters. Because our goal was to provide a parsimonious account of the data, we began with highly constrained versions of the model and freed up parameter constraints when it aided in predicting bias. To provide a statistical evaluation of the utility of the model, we subtracted bias values predicted by the model from participants' data and conducted an ANOVA on these residuals. A useful model would provide a parsimonious account of the data and eliminate or strongly reduce the key interactions with shape.

Figure 2 presents the actual dot locations, the mean responses of participants, and model predictions of responses. In a series of simultaneous fits to the three rounded shapes, an 11-parameter model was found to explain the data well; parameter values are presented in Table 1. This model estimated the four prototype locations for each shape under several constraints. The first was that vertical and horizontal symmetry applied to prototype locations. Accordingly, a single value of $x$ was used for the two leftmost prototypes and a single value of $x$ was used for the two rightmost prototypes in the circle condition. Likewise, a single value of $y$ was used for the two topmost prototypes and a single value of $y$ was used for the two bottommost prototypes for the circle. Thus, the four prototypes of the circle condition were described by just four parameters. For the horizontal ellipse, the $y$-values of
Circle

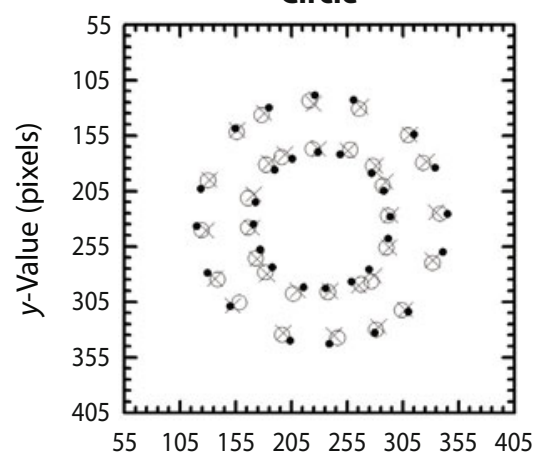

Horizontal Ellipse

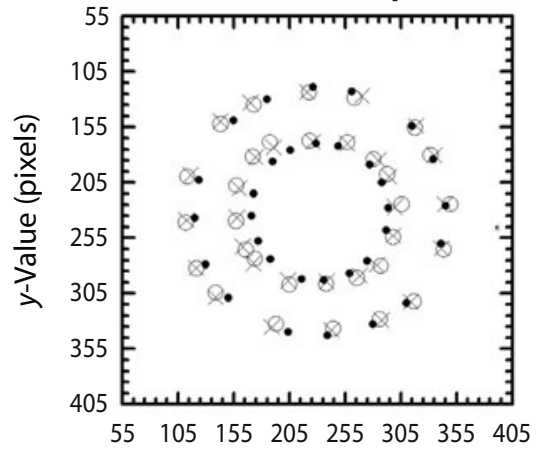

Vertical Ellipse

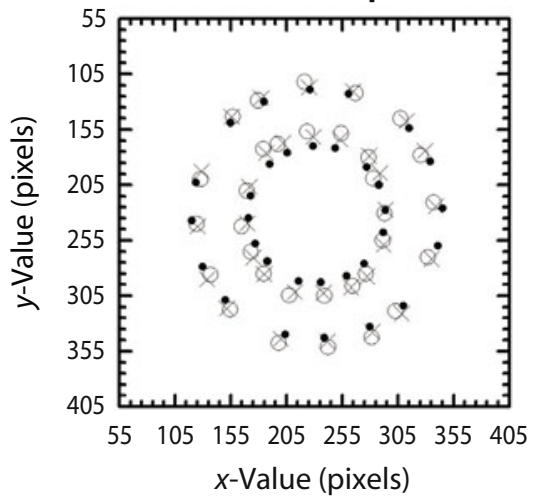

Square
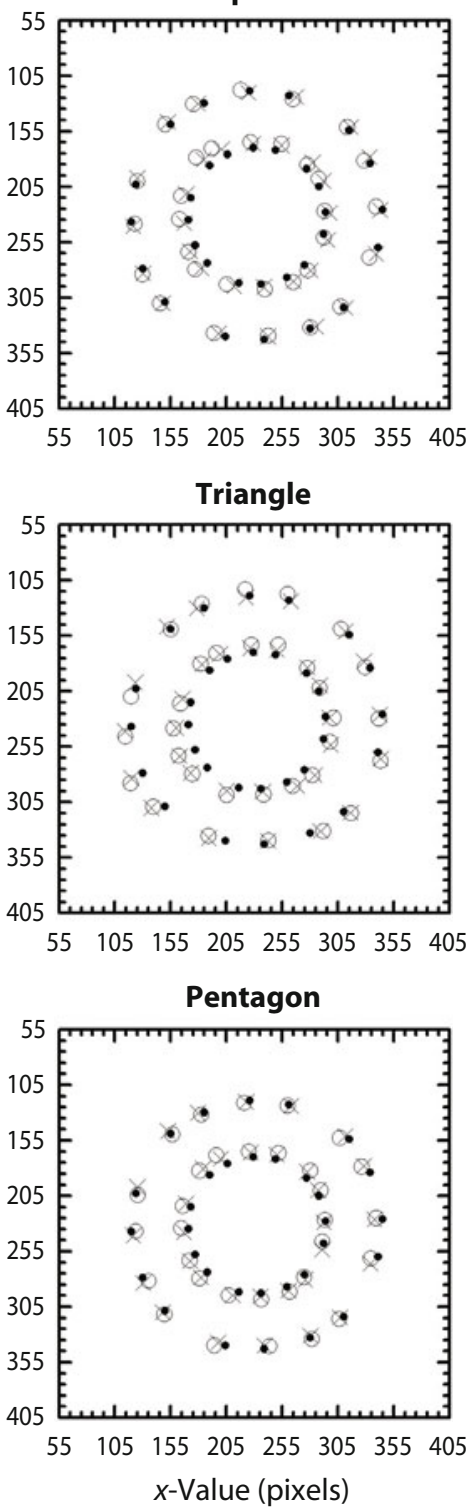

Figure 2. Comparison of estimated locations, actual locations, and model-predicted locations as a function of shape of task field. The small filled circles represent the actual dot locations, the large open circles represent the mean of estimated locations by participants, and the Xs represent the model-predicted locations. 
Table 1

Parameter Values for 11-Parameter Model Fit to Rounded Shape Conditions

\begin{tabular}{lcccccccccc}
\hline Shape & $w$ & $c$ & $x_{1}$ & $y_{1}$ & $x_{2}$ & $y_{2}$ & $x_{3}$ & $y_{3}$ & $x_{4}$ & $y_{4}$ \\
\hline $\begin{array}{l}\text { Circle } \\
\begin{array}{c}\text { Horizontal } \\
\text { ellipse }\end{array}\end{array}$ & $\mathbf{0 . 7 9 6}$ & $\mathbf{0 . 0 5 5}$ & $\mathbf{2 9 6}$ & $\mathbf{1 5 4}$ & $\mathbf{1 6 1}$ & 154 & 161 & $\mathbf{3 0 5}$ & 296 & 305 \\
$\begin{array}{c}\text { Vertical } \\
\text { ellipse }\end{array}$ & 0.873 & 0.055 & $\mathbf{3 4 3}$ & 154 & $\mathbf{6 2}$ & 154 & 62 & 305 & 343 & 305 \\
\hline
\end{tabular}

Note- $w$, fine-grain weighting parameter (Equation 1); $c$, sensitivity parameter (Equation 2); $x$, horizontal value (increasing left to right); $y$, vertical value (increasing top to bottom); subscripts for $x$ and $y$ indicate which prototype they refer to; boldfaced values are free to vary; italicized values were constrained to be equal to a boldfaced value.

the prototypes were constrained to be equal to the $y$-values for the corresponding prototypes of the circle, but two additional $x$-values were estimated (one for leftmost prototypes and one for rightmost prototypes). For the vertical ellipse, the $x$-values of the prototypes were constrained to be equal to the $x$-values for the corresponding prototypes in the circle condition, but two additional $y$-values were estimated for topmost and bottommost prototypes. We modeled prototype recruitment using Equation 2, with only one estimated parameter $(c)$. In fitting this portion of the model, we found that the best fit was achieved by using the circle prototype values for all three shape conditions. Finally, we estimated two values of the finegrain weighting parameter $(w)$, one for the circle and one for the two ellipses. When this 11-parameter model was fit to the 192 data points, the explained variance in bias scores was quite high $\left(R^{2}=.833\right)$. When residuals from this model were entered into an ANOVA, three of the five significant interactions with shape were rendered nonsignificant, including the two largest interactions $\left(F_{\mathrm{s}}>30\right)$. The remaining three-way and four-way interactions were reduced in magnitude by more than one half. Thus, the analysis of residuals implies that the model provided a good account of the differences in bias resulting from the shape manipulation in the rounded shape condition.

In a series of simultaneous fits to the three angular shape conditions, a 10-parameter model was found to explain the data well; parameter values are presented in Table 2. In this model, four prototype locations for each shape were estimated under several constraints. For the square shape, vertical and horizontal symmetry constraints were again applied to prototype locations. Thus, only four parameters were needed to model the four prototypes for the square. The horizontal symmetry constraint was not applied to the triangle condition, because the elongation along the base implied that $x$-values for the two lower prototypes would be more extreme than for the two upper prototypes. Similar possible problems applied to the pentagon shape. Hence, in these conditions, $x$ - and $y$-values were originally left free to vary and only constrained when the drop in $R^{2}$ was not significant. As it turned out, the $x$-values for all four prototypes in the triangle condition were free to vary, but the $y$-values were constrained to be equal to corresponding $y$-values from the square condition. The pentagon shape required no new free parameters for the inferred $x$-values, with two constrained to be equal to values from the square condition and two to values from the triangle condition. The prototype recruitment was modeled using Equation 2, with only one estimated parameter $(c)$. Once again, in fitting this portion of the model, it was found that the best fit was achieved by using the square prototype values for all three shape conditions. Finally, one value of the fine-grain weighting parameter $(w)$ was estimated for all three shapes. When this 10-parameter model was fit to the 192 data points, the explained variance in bias scores was moderately high $\left(R^{2}=.691\right)$. Finally, when residuals from this model were entered into an ANOVA, the two largest interactions were rendered nonsignificant, and the other two were reduced but still significant. Thus, the model provided a reasonable account of the largest differences in bias resulting from the shape manipulation in the angular shape condition.

Table 3 presents an analysis of how the reported models fared against models with different prototype constraints. For the rounded shapes, one critical issue is whether the constraints on $x$ - and $y$-values were reasonable. As shown in Table 3, the increment in $R^{2}$ for the less constrained model was minimal and nonsignificant, despite the large increase in number of parameters. Two competing models are relevant for the angular shapes. As shown in Table 3, a model that uses just three prototypes for the triangle reduces $R^{2}$ significantly, indicating the need for a fourth pro-

Table 2

Parameter Values for the 10-Parameter Model Fit to Angular Shape Conditions

\begin{tabular}{lcccccccccc}
\hline Shape & $w$ & $c$ & $x_{1}$ & $y_{1}$ & $x_{2}$ & $y_{2}$ & $x_{3}$ & $y_{3}$ & $x_{4}$ & $y_{4}$ \\
\hline Square & $\mathbf{0 . 8 8 1}$ & $\mathbf{0 . 0 4 5}$ & $\mathbf{3 2 5}$ & $\mathbf{1 3 3}$ & $\mathbf{1 4 0}$ & 133 & 140 & $\mathbf{3 1 9}$ & 325 & 319 \\
Triangle & 0.881 & 0.045 & $\mathbf{2 8 4}$ & 133 & $\mathbf{1 2 7}$ & 133 & $\mathbf{4 6}$ & 319 & $\mathbf{3 6 2}$ & 319 \\
Pentagon & 0.881 & 0.045 & 284 & 133 & 140 & 133 & 140 & 319 & 284 & 319 \\
\hline
\end{tabular}

Note- $w$, fine-grain weighting parameter (Equation 1 ); $c$, sensitivity parameter (Equation 2); $x$, horizontal value (increasing left to right); $y$, vertical value (increasing top to bottom); subscripts for $x$ and $y$ indicate which prototype they refer to; boldfaced values are free to vary; italicized values were constrained to be equal to a boldfaced value. 
Table 3

Comparison of Model Fits

\begin{tabular}{lccl}
\hline $\begin{array}{c}\text { Shape } \\
\text { Condition }\end{array}$ & $R^{2}$ & Parameters & \\
\hline Rounded & .833 & 11 & Base model of Table 1 with four prototypes \\
Rounded & .857 & 27 & Base model nested within this model with four prototypes but no constraints on $x$ - and $y$-values \\
Angular & .692 & 12 & Base model of Table 2 with four prototypes and two added parameters \\
Angular & .680 & 11 & Nested-within-base model with three prototypes for triangle \\
Angular & .701 & 14 & Base model nested within this model with five prototypes for pentagon \\
\hline
\end{tabular}

Note $-R^{2}$ values reflect the proportion of variance in bias explained by the given model; $F$ tests based on the change in $R^{2}$ from the base model showed no significant increment in variance explained for any of the alternative models and a significant decrement for the model that included only three prototypes for the triangle condition; the base model for the angular data added two nonsignificant parameters to the one reported in the text in order to achieve proper nesting of models for statistical tests.

totype. Also illustrated is a nonsignificant increment in $R^{2}$ for a model in which an additional prototype was fit to the pentagon condition, supportive of the four-prototype model in this condition. These supplemental analyses provide additional support for the models described in Tables 1 and 2.

\section{DISCUSSION}

Our experimental results were consistent with the idea that the shape of the surrounding task field can bias estimation through systematic shifts of geometrically based category prototypes. Because the set of targets for each shape was exactly the same, these effects cannot be attributed to changes in the target distribution. To clarify the bases for these effects, Figure 1 superimposes the prototype values inferred from our modeling (see Tables 1 and 2 ) onto the shapes we used. For the rounded shapes, the inferred prototype locations showed a close correspondence to the relative elongation along the $x$ - and $y$-axes. The prototype locations inferred for the circle were consistent with previous research (Huttenlocher et al., 1991). Widening the shape by creating a horizontal ellipse resulted in large shifts of the prototype locations along the $x$-axis to reflect the extremity of the spatial configuration along this axis. However, modeling demonstrated that the $y$-coordinates for the prototypes did not shift relative to those for the circle. Corresponding conclusions apply to prototype locations within the vertical ellipse.

The angular shape manipulation shows a similar tendency of prototypes to shift with elongation along the $x$ axis. This was most notable when comparing $x$-values of inferred prototypes along the base of the triangle to those along the top of the triangle. A focus of the angular manipulation was to determine whether the number of prototypes might change with spatial configuration, consistent with an object-based orientation. However, there was no advantage to modeling a fifth prototype for the pentagon frame, and there was a distinct disadvantage to modeling only three prototypes for the triangle frame. The results were more consistent with the use of a viewer-based frame of reference, according to which the left-right and up-down distinctions constitute the bases for creating spatial categories. In general, prototype locations in Figure 1 exhibit a leftward bias relative to the center of the shape. We believe this may be tied to a general leftward response bias, perhaps due to the use of the mouse for responding.
Because our focus was on the number and relative locations of prototypes, we did not pursue alternative ways to model this leftward bias, but instead let it be reflected in the prototype locations.

The regularity of these results and the applicability of modeling procedures imply that several related questions could be successfully investigated using these procedures. For example, what would happen if the ellipse had its major diagonal along the $45^{\circ}$ axis? Predictions would differ based on frame of reference (viewer based or object based), and these could be tested using modeling procedures. Because Fitting et al. (2005) found that the viewer-based frame of reference was abandoned for a cue-based frame of reference when the shape was rotated on a majority of trials, we suspect that a similar shift to an object-based frame of reference might be found if the orientation to the shape was systematically varied. Manipulation of the complexity of the figures might also be very important, because distinct "corridors" or "rooms" would likely lead to a more object-based frame of reference and consequent changes in number and location of prototypes. Such questions are amenable to experimental exploration using these types of modeling procedures.

\section{AUTHOR NOTE}

Correspondence concerning this article should be addressed to D. H. Wedell, Department of Psychology, University of South Carolina, Columbia, SC 29208 (e-mail: wedell@ sc.edu).

\section{REFERENCES}

Cheng, K. (1986). A purely geometric module in the rat's spatial representation. Cognition, 23, 149-178.

Fitting, S., WedEll, D. H., \& AlleN, G. L. (2005). Memory for spatial location: Influences of environmental cues and task field rotation. In A. G. Cohn \& D. M. Mark (Eds.), COSIT, LNCS 3693 (pp. 459-474). Berlin: Springer.

Haun, D. B. M., Allen, G. L., \& Wedell, D. H. (2005). Bias in spatial memory: A categorical endorsement. Acta Psychologica, 118, 149-170.

Hermer, L., \& Spelke, E. S. (1994). A geometric process for spatial reorientation in young children. Nature, 370, 57-59.

Huttenlocher, J., Hedges, L. V., \& Duncan, S. (1991). Categories and particulars: Prototype effects in estimating spatial location. Psychological Review, 98, 352-376.

SPENCER, J. P., \& HUND, A. M. (2002). Prototypes and particulars: Geometric and experience-dependent spatial categories. Journal of Experimental Psychology: General, 131, 16-37.

(Manuscript received May 19, 2006; revision accepted for publication August 16, 2006.) 